\title{
Amidation of phenol derivatives: a direct synthesis of paracetamol
}

\section{(acetaminophen) from hydroquinone}

\author{
Roxan Joncour, ${ }^{a}$ Nicolas Duguet,${ }^{a}$ Estelle Métay, ${ }^{a}$ Amadéo Ferreira ${ }^{b}$ and Marc Lemaire* ${ }^{a}$
}

A direct synthesis of paracetamol (acetaminophen) from hydroquinone has been developed using ammonium acetate as amidating agent. The reaction proceeds in acetic acid at elevated temperature without any metallic catalyst. Under these conditions, paracetamol was obtained with high yield and selectivity (> 95\%). The reaction has also been carried out on the multi-gram scale (44 $\mathrm{g}$ of hydroquinone) and a potential process has been proposed based on the recycling of solvent and by-products. This amidation protocol has also been extended to other phenol derivatives.

\section{Introduction}

$\mathrm{N}$-Arylamides are ubiquitous in biologically active molecules and are widely encountered in pharmaceuticals (e.g., atorvastatin, imatinib) and agrochemicals. Among the plethora of synthetic methods developed so far, ${ }^{1}$ the direct creation of the $\mathrm{C}-\mathrm{N}$ bond through cross-coupling of arylhalides $(\mathrm{I}, \mathrm{Br}, \mathrm{Cl}$ ) or pseudohalides (OTf, OTs, OMs,...) with primary or secondary amides offers one of the best options in terms of versatility. These amidation methods are mainly catalyzed by palladium (Buchwald-Hartwig cross-coupling) ${ }^{2}$ and copper (Ullmann-Goldberg condensation). ${ }^{3}$ Alternatively, $\mathrm{N}$ arylamides could be prepared through Chan-Lam-Evans crosscoupling using arylboronic derivatives under oxidative conditions (Scheme 1). ${ }^{4}$ Although these methods are robust and wellestablished, it is necessary to incorporate the leaving group beforehand on the aromatic coupling partner which finally ends up in the waste. Consequently, the atom-economy of the overall process remains modest.

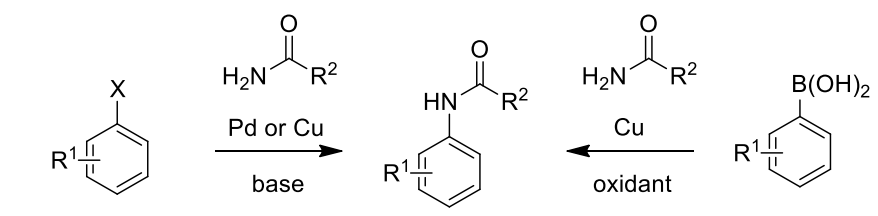

$\mathrm{X}=\mathrm{Cl}, \mathrm{Br}, \mathrm{I}, \mathrm{OTf}, \mathrm{OTs}, \ldots$

Scheme 1. Main routes for direct amidation.

$N$-Acetyl-para-aminophenol (APAP), commonly known as paracetamol or acetaminophen, is a representative of the $\mathrm{N}$ arylamide class. This drug is one of the most consumed worldwide with a global production of more than 100,000 tons per year. ${ }^{5}$ However, the relative simplicity of its structure makes this molecule a low added value compound with an estimated production cost of 3-4 euros/Kg. ${ }^{6}$ Hence, the paracetamol synthesis requires a limited number of steps with excellent efficiencies and high atomeconomies. In this context, the aforementioned cross-coupling technologies have not found their place yet. Over the last century, many routes have been explored for the paracetamol production but all those which have emerged industrially are based on the acetylation of para-aminophenol (PAP) as final stage (Scheme 2). That is the reason why, the access to this key intermediate has been the focus of numerous studies.

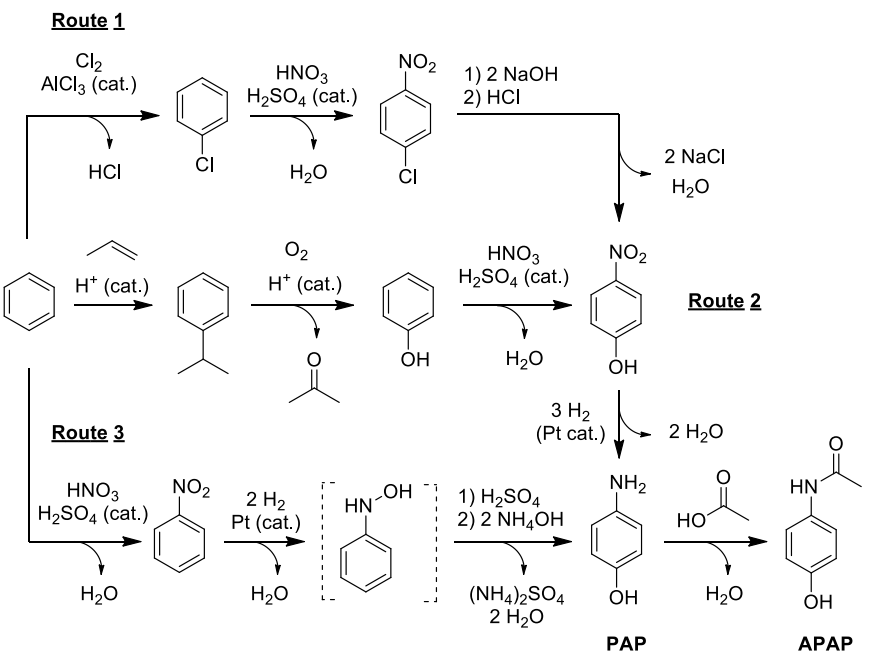

Scheme 2. Commercial routes for paracetamol production.

First, para-aminophenol could be obtained by nitration of chlorobenzene, followed by hydrolysis under alkaline conditions (and subsequent acidification) and hydrogenation of the corresponding para-nitrophenol (Scheme 2, Route 1). ${ }^{7}$ This approach requires 3 steps from chlorobenzene ( 4 from benzene) and generates many salts which induce a modest atom economy of about $38 \% .^{8}$ The alternative approach in which para-nitrophenol is prepared by direct nitration of phenol also provides para-aminophenol in 4 steps from benzene, but provides a process with an improved atom economy of about $54 \%$ (Scheme 2, Route 2). In both cases, the nitration leads to a significant proportion of unwanted ortho-isomer that could not be avoided. A second possibility involves the hydrogenation of nitrobenzene to phenylhydroxylamine that is transformed under acidic conditions to the desired paraaminophenol via a Bamberger rearrangement (Scheme 2, Route 3). ${ }^{9}$ Although more attractive than route 1 , this last route suffers from moderate selectivity (aniline is obtained as by-product) and the generation of large quantities of sulphate salts that reduces the overall atom-economy (about $52 \%$ based on benzene). ${ }^{8}$ Several attempts have been made to substitute the corrosive and polluting sulphuric acid by solid acids but low selectivities are generally obtained..$^{10}$ Therefore, there is still an incentive to develop a greener 
process for the paracetamol production.

One of the most promising routes in term of atom-economy is the direct amidation of hydroquinone with acetamide since it generates only water as by-product. It should be noted that hydroquinone is industrially produced by direct oxidation of phenol which is also used as starting material for the production of para-nitrophenol (Scheme 2, Route 2). Gopinathan et al. have shown that hydroquinone could react with acetamide at elevated temperature $\left(280-300^{\circ} \mathrm{C}\right)$ in the presence of solid acid catalysts such as zeolite $\beta$ or a heteropolyacid. ${ }^{11}$ Under these conditions, paracetamol was obtained with $86 \%$ yield. Qiu et al. have also used a H-SZM-5 zeolite catalyst at $300^{\circ} \mathrm{C}$, confirming the difficulty to activate this reaction probably due to the relatively poor nucleophilicity of acetamide. $^{12}$

We have previously described the nucleophilic substitution of phenol derivatives - including hydroquinone - with alcohols to give access to aromatic ethers using Brønsted or Lewis acid catalysis at $115^{\circ} \mathrm{C} .{ }^{13}$ As this nucleophilic substitution could also be carried out using aniline, we envisioned that the reaction could work with acetamide. Unfortunately, all attempts to incorporate the acetamido group failed under our conditions. ${ }^{14}$ We now report here an alternative method for the direct synthesis of paracetamol from hydroquinone using ammonium acetate as amidating agent. ${ }^{15}$ Both starting materials are relatively cheap and available in bulk quantities. The proposed route gives access to paracetamol without salt production and water as by-product.

\section{Results and discussion}

Inspired by the early work of Bean and Donovan, ${ }^{16}$ who described the amination of hydroquinone using a mixture of diammonium phosphate and ammonia at $200^{\circ} \mathrm{C}$, we focussed our attention on the use of ammonium acetate for the amidation of hydroquinone. It was anticipated that the production of para-aminophenol in the presence of the acetate counter-anion would lead to the desired acetamido functionality (Scheme 3).

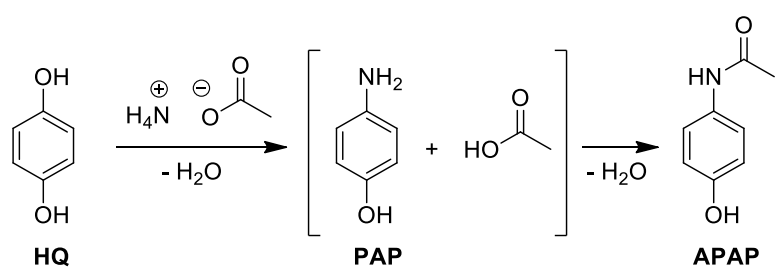

Scheme 3. Proposed route for paracetamol.

Thus, hydroquinone (HQ) was treated with a stoichiometric amount of ammonium acetate at $180^{\circ} \mathrm{C}$ for 15 hours under solventfree conditions. ${ }^{17}$ Satisfyingly, a $63 \%$ conversion of hydroquinone to a 91:9 mixture of APAP and PAP was achieved with $68 \%$ selectivity (Table 1, Entry 1). When heated at $220^{\circ} \mathrm{C}$, the reaction was almost complete $(98 \%)$ but the overall selectivity dramatically dropped to $42 \%$ (Table 1, Entry 2). This could be explained by the formation of diarylamines that have been identified by LC-MS (Figure 1). These by-products are probably formed by the reaction of paraaminophenol with hydroquinone and further acetylation and/or acetamidation. We hypothesized that diarylation occurs when working under high concentration. Therefore, hydroquinone was treated with 3.3 equivalents of ammonium acetate at $180^{\circ} \mathrm{C}$. Under these conditions, the selectivity increased to $94 \%$ (Table 1, Entry 3) and was further improved to $>95 \%$ when working with 10 equivalents (Table 1, Entry 4). Meanwhile, the conversion of hydroquinone slightly dropped from 63 to $48 \%$ and the ratio between APAP and PAP remained relatively constant (around 85:15). Finally, the increase of the temperature to $200^{\circ} \mathrm{C}$ slightly improved the conversion $(65 \%)$ and further increase to $220^{\circ} \mathrm{C}$ permitted to reach $98 \%$ conversion. However, acetylation of para-aminophenol was incomplete (79-85\%) in both cases (Table 1, Entries 5 and 6).

Table 1. Optimization of reaction conditions. ${ }^{\text {[a] }}$

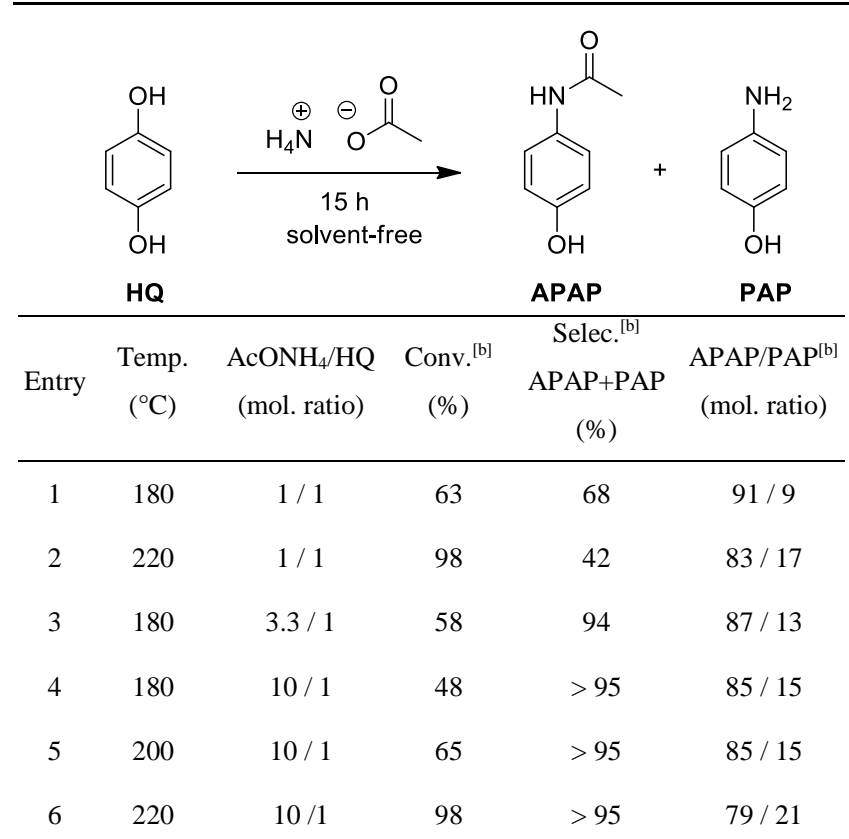

[a] Reaction conditions: Hydroquinone (40-100 mmol), $\mathrm{AcONH}_{4}, 15$ hours. ${ }^{[\mathrm{b}]}$ Determined by HPLC.
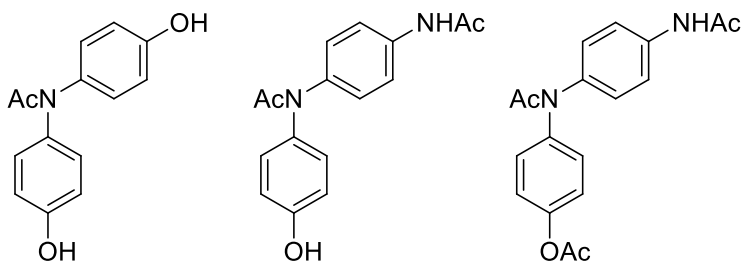

Figure 1. Diarylamine by-products detected by LC-MS.

In order to increase the acetylation of para-aminophenol to paracetamol, we then turned our attention to the use of acetic acid as acylating agent. Thus, hydroquinone was reacted under the previously optimized conditions in the presence of acetic acid (5 equiv.) at $220^{\circ} \mathrm{C}$ for 15 hours. Pleasingly, hydroquinone was converted with $96 \%$ and the global selectivity reached $>95 \%$ (Table 2, Entry 1). More importantly, neither para-aminophenol nor its diarylamine derivatives could be detected under these conditions confirming its full acetylation to paracetamol. However, the use of a large excess of ammonium acetate still compromises this route for potential industrial application. Decreasing its amount to 2 and 1.2 equivalent led to lower conversions ( $85 \%$ and $76 \%$, respectively) while the selectivity and the ratio between APAP and PAP were hardly altered (Table 2, Entries 2 and 3). The same observations 
were made when using 2 equivalents of ammonium acetate and only one equivalent of acetic acid at $180^{\circ} \mathrm{C}$ (Table 2, Entry 4). Finally, a 2:5 mixture of ammonium acetate and acetic acid was selected as a compromise which preserved high selectivity with an acceptable conversion (Table 2, Entry 2).

Table 2. Amidation of hydroquinone in acetic acid. ${ }^{[\mathrm{a}]}$

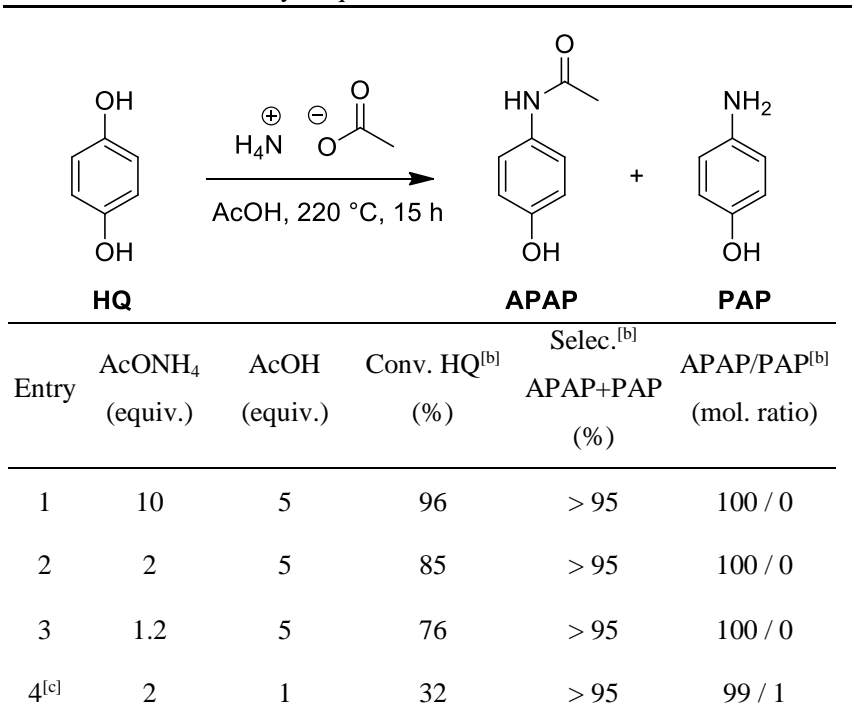

[a] Reaction conditions: Hydroquinone (20-100 mmol), $\mathrm{AcONH}_{4}, 220{ }^{\circ} \mathrm{C}, 15$ hours. ${ }^{[b]}$ Determined by HPLC. ${ }^{[c]}$ Reaction performed at $180{ }^{\circ} \mathrm{C}$ for 15 hours.

From a mechanistic point of view, the reaction of hydroquinone with ammonium acetate could produce para-aminophenol as intermediate which could be directly acetylated to acetyl-paraaminophenol in the presence of acetic acid. In this sequence, the formation of paracetamol is accompanied with the production of two molecules of water (Scheme 4, Route 1). However, an alternative mechanism pathway could not be ruled out under our conditions. Indeed, at elevated temperature, ammonium acetate could be first dehydrated to acetamide and could further react with hydroquinone to produce one molecule of paracetamol along with one molecule of water (Scheme 4, Route 2). This hypothesis has been confirmed by Noyes and Goebel who have shown that ammonium acetate could be dehydrated to acetamide at $220^{\circ} \mathrm{C}$ with $84 \%$ conversion, and the equilibrium could be obtained in less than one hour. ${ }^{18}$

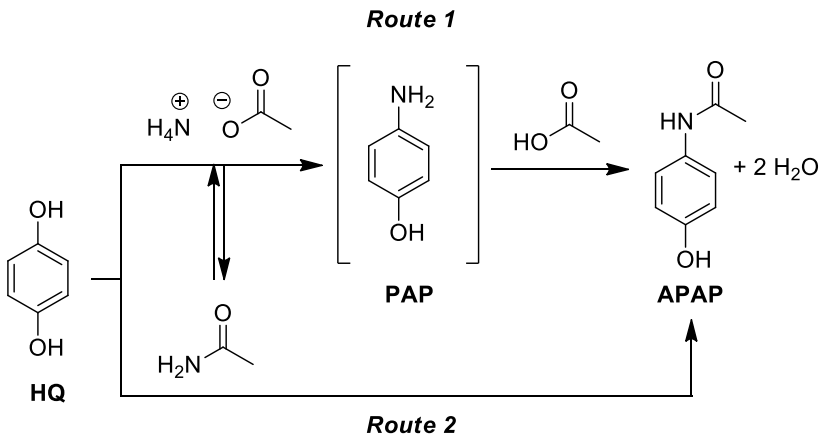

Scheme 4. Potential mechanism pathways.

In order to probe whether either ammonium acetate or acetamide acts as the nucleophile, we carried out the amidation of hydroquinone with 10 equivalents of acetamide for 15 hours at $220^{\circ} \mathrm{C}$. Under these conditions, a conversion of $27 \%$ was obtained with $84 \%$ APAP+PAP selectivity (Table 3, Entry 1). This result indicates that acetamide is a less efficient amidating agent than ammonium acetate and corroborates the findings of Gopinathan ${ }^{11}$ and Qiu. ${ }^{12}$ Indeed, ammonium acetate gave $98 \%$ conversion with > 95\% selectivity using similar conditions (Table 1, Entry 6). Then, hydroquinone was treated with 2 equivalents of acetamide and 5 equivalents of acetic acid to give $56 \%$ conversion and > $95 \%$ selectivity (Table 3, Entry 2). It should be noted that ammonium acetate gave $85 \%$ conversion, proving once again its superiority as amidating agent (Table 2, Entry 2). The presence of acetic acid appears to be essential for the conversion indicating that it could act as both an acylating agent and a catalyst.

Table 3. Amidation of hydroquinone with acetamide. ${ }^{[a]}$

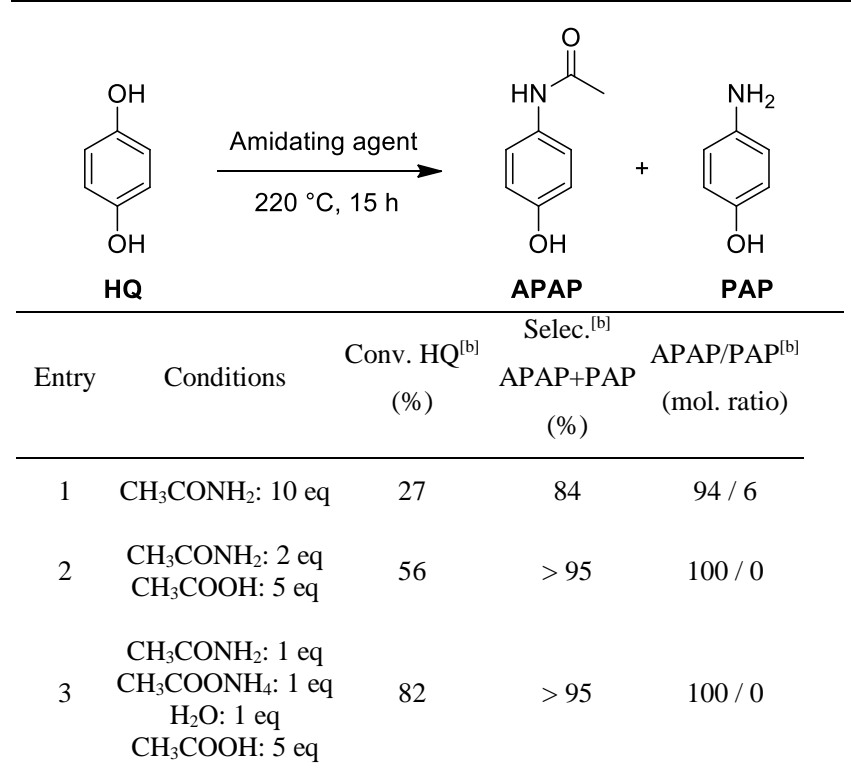

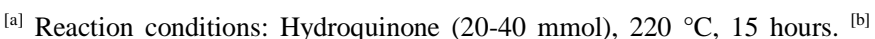
Determined by HPLC.

Finally, the amidation of hydroquinone has been carried out in acetic acid (5 equivalents) using a 1/1/1 mixture of acetamide/ammonium acetate/water in order to simulate the in-situ dehydration of ammonium acetate. In this case, the conversion reached $82 \%$ and the overall selectivity was found superior to $95 \%$ (Table 3, Entry 3). It should be added that no para-aminophenol was be detected. This result indicates that both mechanism pathways could be followed under these conditions but with a predominant character for the route 1 (Scheme 4).

In order to evaluate the potential of this process on larger scale, a batch of paracetamol was prepared using optimized conditions. Thus, $44 \mathrm{~g}(0.4 \mathrm{~mol})$ of hydroquinone was treated with $63 \mathrm{~g}$ of ammonium acetate in $114 \mathrm{~mL}$ of acetic acid at $230{ }^{\circ} \mathrm{C} .{ }^{19}$ After 15 hours, HPLC analysis of the reaction mixture revealed a 93\% conversion of hydroquinone. Acetic acid was distillated from the reaction mixture and recovered with $85 \%$ yield. After cooling, paracetamol precipitates and $53 \mathrm{~g}(88 \%)$ was recovered by filtration. HPLC of the crude product showed that the purity was higher than $99 \%$ (Figure 2). ${ }^{20}$ 


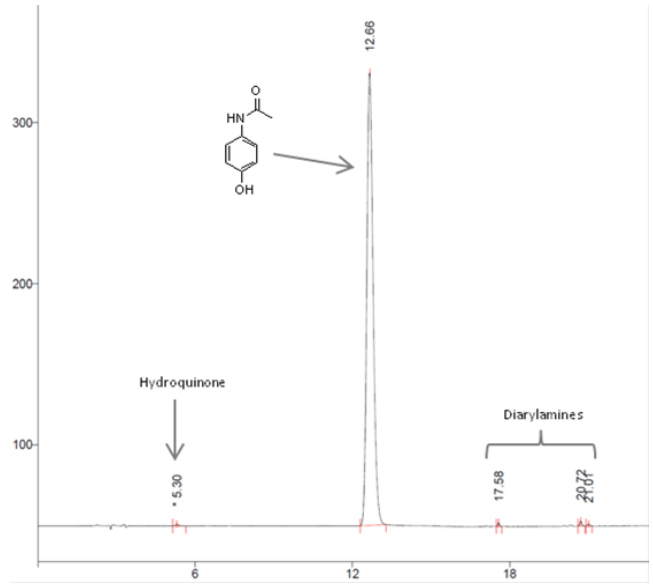

Figure 2. HPLC chromatogram of the crude paracetamol.

The filtrate was also analyzed by HPLC and a 2:1 mixture of acetamide and ammonium acetate was obtained. This mixture could be reused in another batch along with 1 equivalent of ammonium acetate and 1 equivalent of water as previously shown before (Table 3, Entry 3). Thus, we have proven that paracetamol can be prepared from hydroquinone with high yield and selectivity. The recycling of the solvent and the reactant allows the reduction of the practical $\mathrm{E}$ factor of this method. The overall process is depicted on scheme 5.

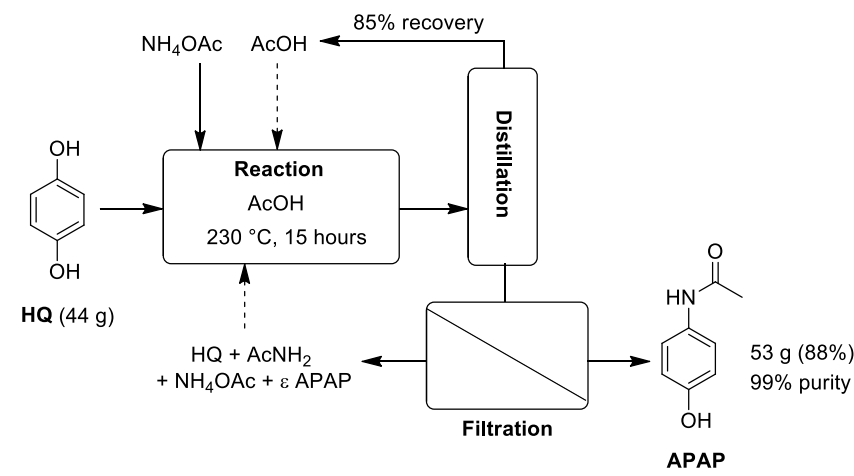

Scheme 5. Amidation of hydroquinone in medium scale.

\section{Scope and limitations}

In order to assess the synthetic utility of this protocol, a range of phenol derivatives was treated with ammonium acetate in the presence of acetic acid at $220^{\circ} \mathrm{C}$ for 15 hours. The results are gathered in Table 4. Di- or tri-hydroxybenzenes were first considered. Resorcinol was converted with $70 \%$ and the corresponding (mono)acetamide was obtained with 50\% isolated yield (Table 4, Entry 1). However, catechol gave only $10 \%$ conversion and a poor 9\% yield (Table 4, Entry 2). Phloroglucinol (1,3,5-trihydroxybenzene) reacted completely giving an insoluble solid that has not been characterized yet. We assumed that this substrate is very reactive towards nitrogenated nucleophiles ${ }^{21}$ and gave a polyaromatic compound. Then, 1-naphthol and 2-naphthol were converted to their corresponding acetamides with 42 and $72 \%$ conversion and with 36 and $56 \%$ isolated yield, respectively (Table 4, Entries 4 and 5). Finally, phenol and other derivatives such as 4chloro- and 4-nitrophenol and 2,6-xylenol were subjected to the same conditions. Unfortunately, these substrates were totally inactive and were recovered unaltered.

Table 4. Amidation of phenol derivatives. ${ }^{[a]}$

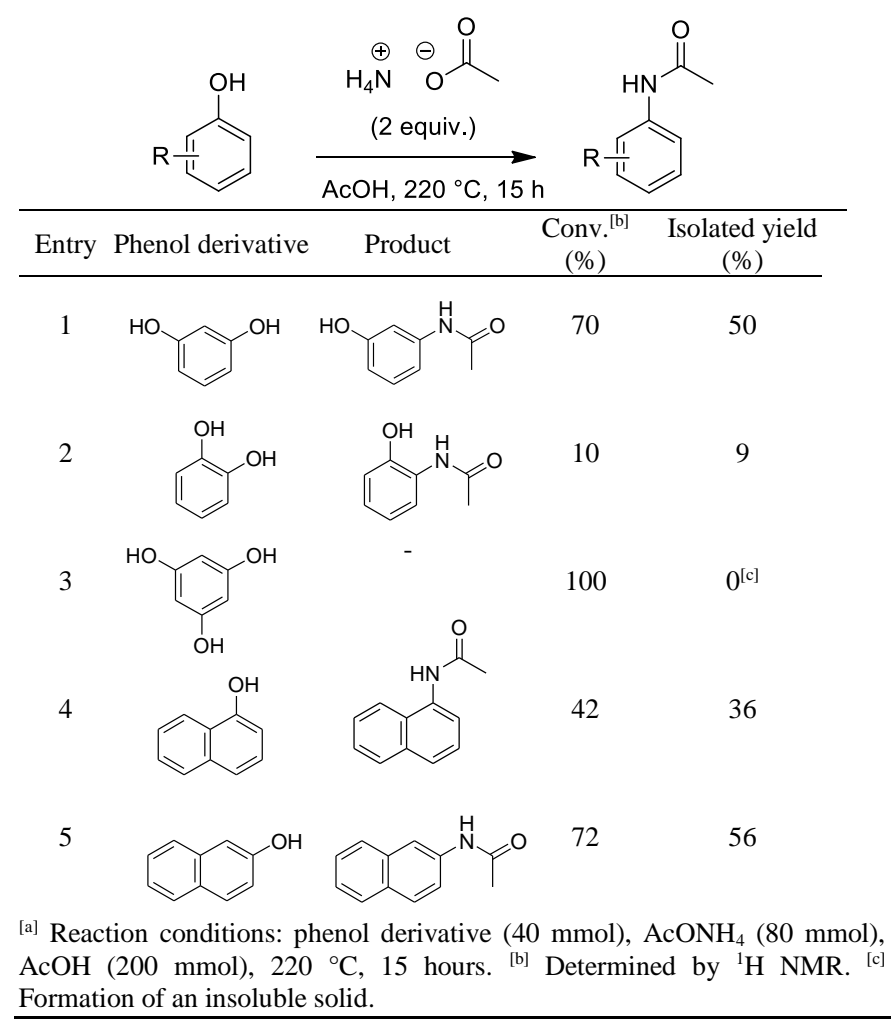

The fact that dihydroxybenzenes are by far more reactive than monohydroxybenzenes has already been noticed in the nucleophilic substitution of phenol derivatives with alcohols. ${ }^{13}$ In that case, we postulated that the mechanism proceeds via a Wheland-type intermediate involving dearomatisation of the benzene or naphthalene rings. In the context of this amidation protocol, the reactivity could also be correlated to the potential ketonization of the phenol derivatives and to the reactivity of the corresponding ketones towards ammonium acetate.

\section{Conclusions}

In conclusion, we have reported a direct synthesis of paracetamol from hydroquinone using ammonium acetate in acetic acid. The reaction proceeds at elevated temperature in the absence of any metallic catalyst and gives excellent selectivity (> 95\%). The reaction has also been demonstrated on the multi-gram scale and a potential process including recycling of acetic acid has been evaluated. In this context, the proposed route gives access to paracetamol without salt production and water as by-product. Furthermore, this amidation protocol has also been extended to a small range of phenol derivatives with low to moderate isolated yields $(9-50 \%)$. Finally, these results gave us insights on the potential intermediates but further studies will be necessary to fully describe the mechanism of this transformation.

\section{Acknowledgments}


The authors would like to thank Minakem and the Association Nationale de la Recherche et de la Technologie (ANRT) for financial support through a CIFRE grant (2011/1190) for R.J.

\section{Notes and references}

${ }^{a}$ Laboratoire de CAtalyse SYnthèse et ENvironnement (CASYEN), Institut de Chimie et Biochimie Moléculaires et Supramoléculaires (ICBMS), CNRS, UMR-5246, Université Claude Bernard Lyon 1, 43 boulevard du 11 novembre 1918, Bât. Curien/CPE, 69622, Villeurbanne, France. E-mail: marc.lemaire.chimie@univ-lyon1.fr; Fax: +33-472-4314-08; Tel: +33-472-43-14-07.

${ }^{b}$ Minakem SAS, 145 chemin des Lilas, 59310 Beuvry-la-Forêt, France.

Electronic Supplementary Information (ESI) available: [calculation of atom-economies, HPLC method, general procedures and characterization data of products]. See DOI: 10.1039/c000000x/

1 a) E. Valeur and M. Bradley, Chem. Soc. Rev. 2009, 38, 606-631. b) C. L. Allen and J. M. J. Williams, Chem. Soc. Rev. 2011, 40, 34053415.

2 For leading references, see: a) W. C. Shakespeare, Tetrahedron Lett. 1999, 40, 2035-2038. b) J. Yin and S. L. Buchwald, Org. Lett. 2000, 2, 1101-1104. c) J. Yin and S. L. Buchwald, J. Am. Chem. Soc. 2002, 124, 6043-6048. d) L. Jiang and S. L. Buchwald, In Metal-Catalyzed Cross-Coupling Reactions, 2nd ed.; A. de Meijere, F. Diederich, Eds.; Wiley-VCH: Weinheim, 2004.

3 For selected reviews, see: a) S. V. Ley and A. W. Thomas, Angew. Chem. Int. Ed. 2003, 42, 5400-5449. b) K. Kunz, U. Scholz and D. Ganzer, Synlett 2003, 15, 2428-2439. c) I. P. Beletskaya and A. V. Cheprakov, Coord. Chem. Rev. 2004, 248, 2337-2364. d) F. Monnier and M. Taillefer, Angew. Chem. Int. Ed. 2009, 48, 6954-6971.

4 For recent reviews, see: a) K. Sanjeeva Rao and T.-S. Wu, Tetrahedron 2012, 68, 7735-7754. b) J. X. Qiao and P. Y. S. Lam, Synthesis 2011, 829-856. c) D. M. T. Chan and P. Y. S. Lam, In Boronic Acids; D. G. Hall, Ed.; Wiley-VCH: Weinheim, 2005; pp 315-361.

5 China and India are the two main producers of paracetamol with more than $110,000-115,000$ tons per year to which should be added the production of other countries in the Asia-Pacific region. The rest of the production comes from the U.S. for an approximate 30,00035,000 tons per year. The production of paracetamol in Europe ceased in 2009.

6 Accurately estimating the production cost of paracetamol is not a simple task as it depends on the route chosen by the manufacturer. Notably, the main differences lie in the selected starting material and the selected technology for the reduction $\left(\mathrm{H}_{2}\right.$ versus $\left.\mathrm{Fe} / \mathrm{HCl}\right)$ of either para-nitrophenol or nitrobenzene.

7 For recent literature on the platinum-catalysed hydrogenation of nitrophenol, see: a) M. Takasaki, Y. Motoyama, K. Higashi, S.-H. Yoon, I. Mochida and H. Nagashima, Org. Lett. 2008, 10, 16011604. b) M. Li, L. Hu, X. Cao, H. Hong, J. Lu and H. Gu, Chem. Eur. J. 2011, 17, 2763-2768. c) K. Xu, Y. Zhang, X. Chen, L. Huang, R. Zhang and J. Huang, Adv. Synth. Catal. 2011, 353, 1260-1264. d) F. Cárdenas-Lizana, C. Berguerand, I. Yuranov and L. Kiwi-Minsker, J. Catal. 2013, 301, 103-111.

8 See Supplementary Information for the calculation of theoretical atom-economies.
9 For recent literature using sulphuric acid, see: a) D. C. Caskey and D. W. Chapman, US 4,571,437, 1986, to Mallinckrodt. b) C. V. Rode, M. J. Vaidya, and R. V. Chaudhari, Org. Process Res. Dev. 1999, 3, 465-470. c) C. V. Rode, M. J. Vaidya, R. Jaganathan and R. V. Chaudhari, Chem. Eng. Sci. 2001, 56, 1299-1304. d) J. M. Nadgeri, N. S. Biradar, P. B. Patil, S. T. Jadkar, A. C. Garade and C. V. Rode, Ind. Eng. Chem. Res. 2011, 50, 5478-5484.

10 For recent literature using solid acid catalysts, see: a) R. V. Chaudhari, S. S. Divekar, M. J. Vaidya and C. V. Rode, US 6,028,227, 2000, to CSIR. b) T. Komatsu and T. Hirose, Appl. Cat. A: Gen. 2004, 276, 95-102. c) S. Wang, Y. Ma, Y. Wang, W. Xue and X. Zhao, J. Chem. Technol. Biotechnol. 2008, 83, 1466-1471. d) P. Liu, Y. Hu, M. Ni, K. You and H. Luo, Catal. Lett. 2010, 140, 6568. e) S. Wang, Y. Wang, Y. Gao and X. Zhao, Chin. J. Catal. 2010, 31, 637-644. f) A. Deshpande, F. Figueras, M. Lakshmi Kantam, K. Jeeva Ratnam, R. Sudarshan Reddy and N. S. Sekhar, J. Catal. 2010, 275, 250-256. g) S. Wang, Y. Jin, B. He, Y. Wang and X. Zhao, Sci. China Chem. 2010, 53, 1514-1519.

11 S. Gopinathan, C. Gopinathan, J. Kuruvilla, S. A. Pardhy and P. Ratnasamy, US 5,856,575, 1999, to CSIR.

12 J. Qiu, J.-G. Wang and J.-X. Dai, Huaxue Shijie 2010, 51, 606-608.

13 C. Cazorla, E. Pfordt, M.-C. Duclos, E. Métay and M. Lemaire, Green Chem. 2011, 13, 2482-2488.

14 Unpublished results.

15 Our results were first published as a patent: Procédé de préparation de dérivés d'acétamidophényle, Fr. Demande 2013, 1359972 A1 20131014.

16 F. R. Bean and T. S. Donovan, US 2,376,112, 1945, to Eastman Kodak Company.

17 Ammonium acetate $\left(\mathrm{mp}=114^{\circ} \mathrm{C}\right)$ is melted at such temperature and therefore can act as both reagent and solvent.

18 W. A. Noyes and W. F. Goebel, J. Am. Chem. Soc. 1922, 44, 22862295.

19 The scale-up was carried out at $230{ }^{\circ} \mathrm{C}$ in order to obtain a higher conversion of hydroquinone. Indeed, a low concentration of hydroquinone facilitates the precipitation of the product from the reaction mixture and furnishes a higher purity of paracetamol.

20 See Supplementary Information for detailed HPLC conditions.

21 Phloroglucinol reacts smoothly with hydroxylamine at room temperature to give the corresponding trioxime, see: J. C. Bottaro, R. Malhotra and A. Dodge, Synthesis 2004, 499-500. 